\title{
Myeloid sarcoma presenting as a recurrent, multifocal nerve root entrapment syndrome
}

\author{
Wiebe C. Verra · Tom J. Snijders · Tatjana Seute • \\ K. Sen Han · H. Karel Nieuwenhuis · Geert Jan Rutten
}

Received: 27 April 2008/Accepted: 4 August 2008/Published online: 19 August 2008

(c) The Author(s) 2008. This article is published with open access at Springerlink.com

\begin{abstract}
Background Myeloid sarcoma is an extramedullary manifestation of haematologic malignancy, most commonly acute myeloid leukemia (AML), which can cause neurological symptoms. Case description A 45-yearold male with a history of AML presented with a lumbosacral nerve root entrapment syndrome followed by cauda equina compression, but without systemic signs of $\mathrm{AML}$ recurrence. MRI showed a mass compressing the spinal cord at level L5-S2. After surgically removing the tumour pathologic examination yielded a myeloid sarcoma. Combined chemotherapy and radiation therapy followed. Five months later the patient developed a thoracal (Th10-Th11) radiculopathy due to a relapse of the myeloid sarcoma, followed by C8-Th1-radiculopathy caused by leptomeningeal spread. Conclusion This case forms the first description of recurrent, multifocal and progressive radiculopathy due to myeloid sarcoma. This diagnosis should be considered in patients with radiculopathy with previous haematological malignancy and/or signs or symptoms of such disease; the
\end{abstract}

W. C. Verra · T. J. Snijders $(\bowtie) \cdot$ T. Seute

Department of Neurology, Rudolf Magnus Institute of

Neuroscience (Internal Address C03.236), University Medical

Center Utrecht, PO Box 85500, 3508 GA Utrecht,

The Netherlands

e-mail: t.j.snijders@umcutrecht.nl

K. S. Han

Department of Neurosurgery, University Medical Center

Utrecht, Utrecht, The Netherlands

H. K. Nieuwenhuis

Department of Haematology, University Medical Center Utrecht,

Utrecht, The Netherlands

G. J. Rutten

Department of Neurosurgery, St. Elisabeth Hospital Tilburg,

Postbus 90151, 5000 LC Tilburg, The Netherlands absence of systemic disease activity does not rule out myeloid sarcoma.

Keywords Myeloid sarcoma - Granulocytic sarcoma . Chloroma $\cdot$ Nerve root entrapment · Radiculopathy . Acute myeloid leukemia
Abbreviations
AML Acute myeloid leukemia
CNS Central nervous system
CSF Cerebrospinal fluid
DLI Donor lymphcyte infusion
GVHD Graft versus host disease

\section{Introduction}

Myeloid sarcoma (also known as granulocytic sarcoma or chloroma) is a rare, malignant, and extramedullary solid tumour, usually a localisation of acute myeloid leukemia (AML). Its manifestations are diverse including several types of central nervous system (CNS) involvement. Considering the diagnosis of myeloid sarcoma at an early stage is important because of the therapeutic options in this potentially devastating disease. It is rare for the clinical manifestation of a myeloid sarcoma to include signs of nerve root entrapment. In the current case we present a patient with myeloid sarcoma who has a multifocal, recurrent, and progressive radiculopathy.

\section{Case}

A 45-year-old Caucasian male visited the Emergency Room because of progressive back pain radiating to the 
right leg. The pain exacerbated by coughing or sneezing. $\mathrm{He}$ also experienced problems with walking. There were no complaints of micturition.

The patient's history included AML, first diagnosed 2.5 years before the onset of the current symptoms. The AML was classified according to the French-AmericanBritish classification as M4, corresponding to an acute myelomonoblastic leukemia with maturation, with an inversion of chromosome 16 . The treatment consisted of chemotherapy (cytarabine/daunorubicin). Sixteen months after the first diagnosis of AML the patient suffered from a relapse. He achieved complete remission after induction therapy with amsacrine and cytarabine followed by nonmyeloablative allogeneic stem cell transplant from his HLA-identical brother after a low dose total body irradiation/fludarabine conditioning regimen.

At neurological examination in the Emergency Room the patient had difficulty walking on his right heel. Aside from this right-sided dorsiflexor weakness, no other muscle weakness was objectified. Further examination revealed a positive straight leg raising (Lasègue's) test and hypoesthesia of the right leg, especially the anterolateral side of the leg and dorsal side of the foot. The right-sided Achilles tendon reflex was decreased compared to the left side, with normal plantar reflexes. Anal sphincter tone was not evidently decreased; peri-anal touch was reported to be 'different' without objectifiable sensory deficits. Based on the positive Lasègue's test, the region of hypoesthesia, and the dorsiflexor weakness it was concluded that the patient was suffering from a lumbosacral radiculopathy (sciatica), best compatible with the right L5 or S1 nerve root. Although the examination of sensory functions contained indications of compression of the cauda equina, this could not be objectified.

MRI of the lumbar spine was performed to evaluate the cause of the suspected compression, revealing an intraspinal, extradurally diffusely contrast-enhancing mass ventral of the cauda equina (Fig. 1a-c). The mass was located at the level L5-S2, following the trajectory of both $\mathrm{S} 1$ and right $\mathrm{S} 2$ nerve roots with visual compression of the cauda equina. Radiologically, an AML-related mass or a nerve sheath tumour (e.g. Schwannoma, although the multilobar appearance was atypical), was considered.

The day after presentation difficulty with micturition developed. Because of the signs of progressive cauda equina compression, urgent surgical treatment was indicated. Tissue diagnosis was required to guide further therapy due to diagnostic uncertainty about the tumour type. Because of the anatomical location of the tumour a CT guided needle biopsy was not possible.

The neurosurgeon then performed laminectomy with partial resection of the tumour; total resection was impossible due to the lateral extension of the tumour through the neuroforamen. During operation, adhesion of the tumour throughout the right-sided L5 nerve root trajectory was confirmed. The tumour was solid and not green (the typical colour of myeloid sarcomas). The tumour was resected with section of the right-sided L5 radix; because of the size and mass effect of the (very solid) tumour, no decline in motor function was expected with section of this radix. Based on the findings from operation, Schwannoma was considered most likely. There were no post-operative complications and the patient was discharged after 10 days with residual sensory complaints, but with intact micturition, no new muscle weakness and without limitations in his daily activities.

Pathological examination of the obtained tissue yielded an extramedullary relapse of the AML; a myeloid sarcoma. A subsequent bone marrow aspirate did not reveal signs of AML recurrence in the marrow. The patient concurrently received radiotherapy $(2100 \mathrm{cGy}$ in seven fractions of $300 \mathrm{cGy}$ dosed in a $6 \mathrm{~cm}$ track from L4 to S3) as well as chemotherapy with high-dose cytosine arabinoside. A donor lymphocyte infusion (DLI) with $1 \times 10^{7} \mathrm{~T}$-cells per $\mathrm{kg}$ was given to induce a graft-versus-leukemia effect. Follow-up MRI after a month revealed some residual abnormal tissue with diffuse contrast-enhancement. No symptoms of graft-versus-host-disease (GVHD) developed after the DLI.

Five months after being diagnosed with myeloid sarcoma the patient visited the neurologist again, complaining of radiating, shooting pain in his right flank, exacerbated by coughing or sneezing. Physical examination showed hyperesthesia at dermatome Th10-Th11 on the right side without other new abnormalities. The clinical diagnosis of a radiculopathy was made. Spinal MRI revealed a bilateral, intraspinal, extradural, contrast-enhanced mass at Th11Th12 following the course of both Th11 nerve roots into the neuroforamen, slightly compressing the spinal cord (Fig. 2). Because of the patient's history and the radiological appearance, tissue examination was not considered necessary to confirm the presumptive diagnosis of a relapsing myeloid sarcoma. Radiation therapy of the spine was applied at the level of the thoraco-lumbar transition. At first, the symptoms subsided after treatment. A few weeks later a diffusely radiating pain developed in the right shoulder and upper arm, in the approximate area of dermatome C8-Th1. A new MRI of the spine showed diffuse, multifocal staining of the nerve roots at the upper thoracic levels without any new solid masses. Because of the multifocal lesions without a well-circumscribed mass, a lumbar puncture was performed. The CSF contained blast cells, confirming the diagnosis of leptomeningeal (intrathecal) expansion of the AML. The patient was treated with intrathecal cytosine arabinoside resulting in disappearance of the spinal fluid blasts and radiating pain. Because the 
Fig. 1 MRI-examination of the lumbosacral spine of the described patient, revealing (a) a T1-hyperintense intraspinal mass adjacent to vertebrae L5-S1 on sagittal MRI, with (b) homogeneous enhancement after intravenous Gadolinium-injection;

(c) transverse view after gadolinium administration. In the figure legends, white arrows mark the tumour mass and black arrows mark the dural $\mathrm{sac}$
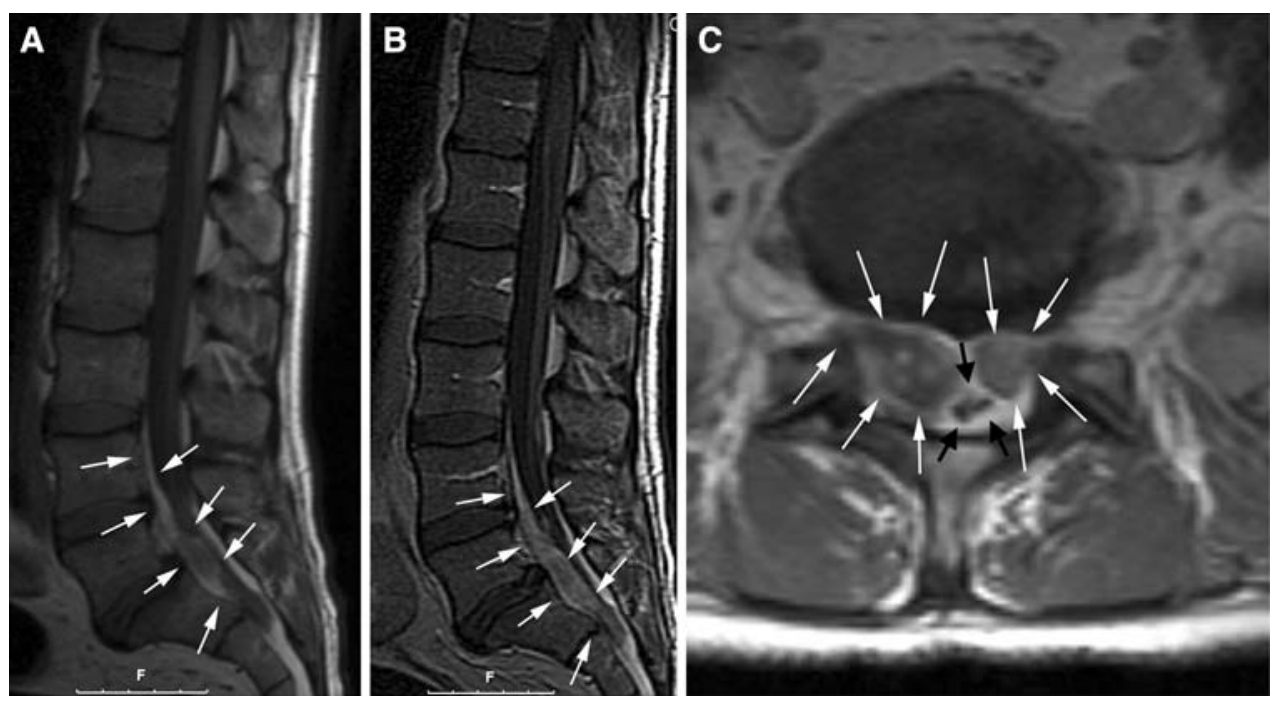

lack of response to the first DLI a second DLI was given with an increased number of donor T-cells $\left(1 \times 10^{8} / \mathrm{kg}\right)$. After three weeks GVHD manifested as generalized erythroderma and mucositis of the mouth. The GVHD, however, was not associated with graft-versus-leukemia effect (i.e. anti-leukemic therapy was unsuccessful), since three months later, he developed progressive loss of vision due to multifocal intracranial AML localizations.

\section{Discussion}

This report describes a patient with recurrent, multifocal nerve root entrapment as the sole manifestation of myeloid sarcoma without systemic signs of leukemia after nonmyeloablative stem cell therapy. To our knowledge this is the first time this has been described.

Myeloid sarcoma is a rare, solid, malignant extramedullary tumour of myeloid cells. It usually occurs in association with AML but it is very rarely seen in other myeloproliferative disorders as well. A total of $2.5-9.0 \%$ of patients with AML develop myeloid sarcoma $[1,2]$. The tumour precedes systemic leukemia in $0.6 \%$ of cases [3]. Myeloid sarcoma is seen more often in men than in women as is generally the case in leukemia [4]. The tumour may appear at any age or localisation, but usually occurs in the subperiost of the vertebra, sternum, orbits and skull. The involvement of the central nervous system (CNS) is rare [5]; myelopathy due to tumour compression is even rarer. Most spinal myeloid sarcomas develop at the thoracic level, followed respectively by the lumbar, sacral and cervical level [6]. In five case reports signs of radiculopathy were objectified $[1,4,5,7,8]$.

Radiculopathies are most commonly caused by a herniated intervertebral disc or degenerative spinal abnormalities,

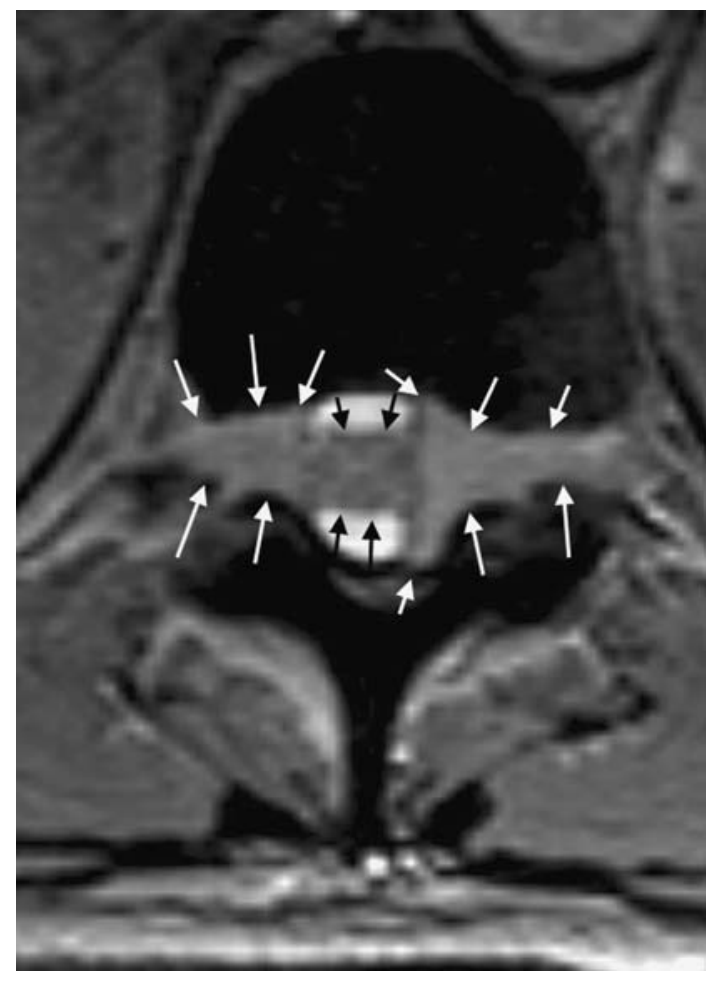

Fig. 2 T2-weighted transverse section at the Th11-Th12 level from cervicothoracal spinal MRI at the time of first recurrence, revealing a bilateral extra-axial mass with periradicular extension into both neuroforamina, causing spinal cord compression. In the figure legend, white arrows mark the tumour mass and black arrows mark the spinal cord

but the differential diagnosis includes many disorders including infections (e.g. Borrelia polyneuroradiculitis), benign tumours (e.g. Schwannoma) and malignancy. A neoplastic cause may be considered based on clues in the history, signs at physical examination or laboratory abnormalities. Diagnostic work-up in patients with radiculopathy 
in whom malignancy is considered should include neuroimaging studies. Abnormalities on CT-scanning are non specific, so MRI is the modality of choice. Myeloid sarcoma is iso-intense on $T_{1}$ - and $T_{2}$-weighted images and generally enhances with gadolinium [9].

In cases where there is no prior history of hematologic malignancy, or in whom the radiologic findings are inconclusive, the investigation of surgically obtained tissue or CSF examination is required for a definite diagnosis. CSF examination can furthermore be used to diagnose intrathecal disease. In our reported case, CSF examination was not performed at the first two episodes of neurological symptoms, although one could argue that a (recurrent) CNS localisation of AML warrants CSF examination. Early intrathecal involvement in AML recurrence thus cannot be excluded in our patient, although this seems unlikely because of the protracted clinical course.

The bone marrow recurrence of AML in this patient was treated with chemotherapy, resulting in complete remission. This was followed by non-myeloablative stem cell transplantation in order to obtain a donor cell mediated graft-versus-leukemia effect. Interestingly, the relapses after the stem cell transplantation and DLI's were extramedullary localized and the bone marrow was free of tumour cells. This suggests that the donor T-cells may have an effect in the bone marrow but are less effective in extramedullary sites like the CNS. Extramedullary relapses after DLI have been described before [10].

The presence of an extramedullary relapse of leukemia is associated with a generally poor response to treatment and short survival. Therapy of the myeloid sarcoma with CNS involvement may include surgical decompression, intravenous and intrathecal chemotherapy, radiation therapy or any combination of these treatments. Since formation of myeloid sarcomas may precede the development of leukemia, systemic treatment is indicated. Treatment of acute spinal cord compression consists of emergency local radiation therapy and corticosteroids; only in cases of diagnostic uncertainty, severe spinal cord compression or contra-indication for radiation (e.g. prior radiation at the same site) surgery is indicated.

In conclusion, the diagnosis of myeloid sarcoma should be considered in patients with radiculopathy with previous haematological malignancy and/or signs or symptoms of such disease; the absence of systemic disease activity does not rule out myeloid sarcoma.

Open Access This article is distributed under the terms of the Creative Commons Attribution Noncommercial License which permits any noncommercial use, distribution, and reproduction in any medium, provided the original author(s) and source are credited.

\section{References}

1. Graham A, Hodgson T, Jacubowski J, Norfolk D, Smith C (2001) MRI of perineural extramedullary granulocytic sarcoma. Neuroradiology 43:492-495. doi:10.1007/s002340000514

2. Neiman RS, Barcos M, Berard C, Bonner H, Mann R, Rydell RE et al (1981) Granulocytic sarcoma: a clinicopathologic study of 61 biopsied cases. Cancer 48:1426-1437. doi:10.1002/1097-0142 (19810915)48:6<1426::AID-CNCR2820480626>3.0.CO;2-G

3. Krause JR (1979) Granulocytic sarcoma preceding acute leukemia: a report of six cases. Cancer 44:1017-1021. doi:10.1002/10970142(197909)44:3<1017::AID-CNCR2820440333>3.0.CO;2-I

4. Mostafavi H, Lennarson PJ, Traynelis VC (2000) Granulocytic sarcoma of the spine. Neurosurgery 46:78-83. doi:10.1097/000 06123-200001000-00016

5. Widhalm G, Dietrich W, Mullauer L, Streubel B, Rabitsch W, Kotter MR et al (2006) Myeloid sarcoma with multiple lesions of the central nervous system in a patient without leukemia. Case report. J Neurosurg 105:916-919. doi:10.3171/jns.2006.105.6.916

6. Landis DM, Aboulafia DM (2003) Granulocytic sarcoma: an unusual complication of aleukemic myeloid leukemia causing spinal cord compression. A case report and literature review. Leuk Lymphoma 44:1753-1760. doi:10.1080/1042819031000104051

7. Balleari E, Panarello S, Capello E, Grosso M, Passalia C, Pitto P et al (2007) Granulocytic sarcoma: an unusual cause of spinal cord compression. Int J Clin Oncol 12:234-237. doi:10.1007/ s10147-006-0645-7

8. Kim FS, Rutka JT, Bernstein M, Resch L, Warner E, Pantalony D (1990) Intradural granulocytic sarcoma presenting as a lumbar radiculopathy. Case report. J Neurosurg 72:663-667

9. Ang P, Virapongse C (1990) Magnetic resonance imaging of spinal intradural granulocytic sarcoma. Magn Reson Imaging 8:95-100. doi:10.1016/0730-725X(90)90218-Q

10. Singhal S, Powles R, Kulkarni S, Treleaven J, Saso R, Mehta J (1999) Long-term follow-up of relapsed acute leukemia treated with immunotherapy after allogeneic transplantation: the inseparability of graft-versus-host disease and graft-versus-leukemia, and the problem of extramedullary relapse. Leuk Lymphoma 32:505-512 